\title{
Efeito do Carbonato de Cálcio na Qualidade da Casca dos Ovos durante a Muda Forçada ${ }^{1}$ José Humberto Vilar da Silva², Valdeci José dos Santos ${ }^{3}$
}

RESUMO - O efeito de fontes de cálcio sobre o desempenho e a qualidade da casca dos ovos, produzidos por 96 poedeiras da linhagem Lohmann - LSL, alojadas às 72 semanas de idade em densidade de duas aves/gaiola, foram avaliados durante e após muda forçada (MF) de 12 dias. O delineamento experimental foi inteiramente ao acaso com quatro tratamentos, cada um com quatro repetições de seis aves. Os tratamentos foram constituídos por: 1 - controle positivo, com ração à vontade em todas as fases do experimento; 2 - MF, sem fonte de cálcio; 3 - MF mais fosfato bicálcico à vontade; e 4 - MF mais carbonato de cálcio à vontade. Durante o segundo ciclo de produção, as aves foram alimentadas com ração comercial e água ad libitum. Foram avaliados os ovos produzidos nos três primeiros dias de MF e em cinco períodos de 30 dias do segundo ciclo de produção. Durante a MF, o uso de carbonato de cálcio resultou em maiores peso e porcentagem de casca dos ovos que o de fosfato bicálcico e o tratamento de MF sem fonte de cálcio. No período subseqüente à MF, o tratamento com carbonato de cálcio melhorou a produção de ovos em relação àquele com fosfato bicálcico e o controle. Estes resultados sugerem que o uso do carbonato de cálcio, durante a muda forçada, foi mais indicado que o de fosfato bicálcico e o tratamento convencional de muda forçada, pela retirada da ração.

Palavras-chave: fontes de cálcio, ovos comerciais, produção de ovos

\section{Effect of Calcium Carbonate on the Egg Shell Quality during the Forced Molt}

ABSTRACT - The effect of calcium source on the performance and egg-shell quality of 96 white-egg layers hens, Lohmann LSL, allotted, at 72 weeks of age, to a density of two layers/pen, were evaluated during and after a forced molt (FM) for 12 days. A completely experimental randomized, with four treatments, each with four replicates of six layers, was used. The treatments were: 1 - positive control, with ad libitum diet during all experimental phases; 2 - FM with no calcium source; 3 - FM with ad libitum dicalcium phosphate; and, 4 - FM with ad libitum calcium carbonate. During the second cycle of production, the birds were full fed commercial diet and water. Egg production in the first three days during the FM and five periods of 30 days of second cycle were evaluated. During FM, the use of calcium carbonate improved the eggshell weight and eggshell percentage than the use of dicalcium phosphate and the treatment of FM without calcium source. In the subsequent period of FM, the use of calcium carbonate showed better egg production than the use of dicalcium phosphate and the control. These results suggest that the use of calcium carbonate during the forced molt was more indicated than the use of dicalcium phosphate and the fast forced conventional practice, by the diet withdraw.

Key Words: calcium source, egg production, marketable eggs

\section{Introdução}

Como o interesse dos avicultores na aplicação da técnica de muda forçada em poedeiras comerciais vem crescendo nos últimos dez anos no Brasil, medidas para o aperfeiçoamento da técnica precisam ser investigadas. Melhor qualidade de casca dos ovos, nos primeiros dias do jejum forçado, é um ponto que deve repercutir diretamente no número de ovos comerciais e no número de ovos perdidos por avaria de casca.

A retirada da ração dos comedouros durante $10 \mathrm{a}$ 12 dias é o método mais simples de induzir a muda em poedeiras e, nos primeiros dias, a produção de ovos declina até a suspensão completa da postura de 4 a 5 dias do início do jejum. A maioria dos ovos produzidos neste período apresenta algum tipo de problema de casca que inviabiliza o seu aproveitamento para a venda. $\mathrm{O}$ aumento do aproveitamento dos ovos produzidos durante o jejum forçado depende dos mesmos fatores que afetam normalmente a qualidade da casca, como linhagem, idade da poedeira, temperatura ambiente, doenças e concentrações dietéticas de cálcio, fósforo e vitamina $\mathrm{D}_{3}$.

$\mathrm{O}$ efeito do cálcio na manutenção da atividade produtiva em poedeiras já é bem documentado na literatura. Poedeiras alimentadas com rações contendo $0,3 \%$ de cálcio suspenderam a postura 7 a 10 dias após o início do fornecimento (URIST, 1959;

\footnotetext{
1 Pesquisa financiada pelo Setor de Avicultura do CAVN/DAP/CFT/UFPB.

2 Departamento de Agropecuária - CFT/UFPB - Bananeiras - PB - CEP 58.220-000. E.mail: jvilar@cft.ufpb.br

3 Aluno do Curso de Graduação em Licenciatura em Técns. Agropecuária/CFT/UFPB.
} 


\section{SILVA e SANTOS}

BELL e SILLER, 1962; e GILBERT, 1969). A possível relação do cálcio com a interrupção da produção de ovos ocorre pela suspensão da liberação das gonadotropinas (MORRIS e NALBANDOV, 1961).

GARLICH e PARKHURST (1982) foram os primeiros a pesquisarem tratamentos para indução de melhor qualidade de casca, durante o período inicial da muda forçada. Os autores perceberam que o uso de cascas de ostras resultou em taxa de declínio da produção mais lenta, menor número de ovos com casca fracas e dobro de ovos comerciais produzidos de 2 a 5 dias do período de jejum.

Segundo BRAKE (1993), o cálcio parece ter papel central na indução da muda, pois, quando o carbonato de cálcio é fornecido como única fonte de nutrientes, as aves prolongam a ovulação durante a muda forçada e mantêm a postura por até quatro dias. Esse autor sugeriu que o cálcio é o primeiro nutriente limitante da ovulação, durante uma muda forçada pela retirada da ração.

Em virtude do balanço negativo de cálcio na fase de muda, em que a produção de ovos é mantida, o fornecimento de fontes de cálcio pode evitar a depleção das reservas de cálcio do tecido medular e do tecido ósseo cortical, podendo promover melhor qualidade da casca do ovo neste período e durante o segundo ciclo de produção.

Dessa forma, o objetivo do presente trabalho foi avaliar o efeito do fornecimento de fontes de cálcio durante a muda forçada sobre a produção e a viabilidade dos ovos no início do jejum e durante o segundo ciclo de produção.

\section{Material e Métodos}

O experimento foi realizado no Setor de Avicultura do Colégio Agrícola André Vidal de Negreiros da Universidade Federal da Paraíba.

Foram utilizadas 96 poedeiras Lohmann Selected Leghorn (LSL), submetidas à muda forçada na $72^{\mathrm{a}}$ semana de idade, pela retirada da ração durante 12 dias, com água ad libitum e fotoperíodo natural. As médias de temperatura e umidade relativa foram, respectivamente, de $24,2^{\circ} \mathrm{C}$ e $73,9 \%$.

As aves foram alojadas, durante o período de produção, em galpão de postura, de $20 \times 9 \mathrm{~m}$, com laterais livre, coberto com telhas de barro em duas águas, sendo utilizadas quatro fileiras de gaiolas numa das laterais do galpão.

Antes da retirada da ração, quatro grupos de aves foram selecionados e distribuídos nos tratamentos pelo peso e pela produção de ovos.

Foi utilizado delineamento inteiramente ao acaso, com quatro tratamentos, cada um com quatro repetições de seis aves.

Os tratamentos aplicados foram: 1 - controle positivo, com ração à vontade em todas as fases do experimento; 2 - muda forçada (MF); 3 - MF com fosfato bicálcico $\left(23 \% \mathrm{Ca}^{+2}\right)$ à vontade; e $4-\mathrm{MF}$ com carbonato de cálcio $\left(38 \% \mathrm{Ca}^{+2}\right)$ à vontade. As fontes de cálcio foram colocadas nos comedouros, logo após a retirada da ração, e a água foi distribuída em bebedouros tipo niple, instalados um por gaiola.

Em virtude do declínio abrupto da postura, a partir do quarto dia de muda, apenas os ovos do dia seguinte à retirada da ração até aqueles produzidos no terceiro dia foram recolhidos para as avaliações.

Após a fase de jejum, as aves passaram por um período de adaptação gradual à ingestão de alimentos, com o fornecimento de cotas crescente de milho moído durante uma semana. Ao atingir $60 \mathrm{~g}$ de ingestão de milho, passou-se a fornecer a ração comercial.

A retomada das coletas nos cinco períodos de 30 dias, posteriores à muda forçada, ocorreu quando as aves completaram 77 semanas de idade, correspondendo à 5a semana após o início da muda, quando a taxa de postura média foi superior a $50 \%$.

As características avaliadas foram produção de ovos, peso médio dos ovos, massa de ovos, peso e porcentagem de casca. Os ovos comerciais foram classificados pela ausência de trincamento e avaria de casca, enquanto os ovos perdidos incluíram todos aqueles sem casca ou com casca fina ou os que apresentaram falhas que impediam a sua comercialização. Os ovos caídos embaixo das gaiolas foram contados e recolhidos diariamente, sendo considerados também nas estimativas.

A porcentagem de casca foi estimada após quebra, pesagem e secagem das cascas em estufa a $105^{\circ} \mathrm{C}$, durante um mínimo de 4 horas, utilizando-se todos os ovos produzidos nos últimos quatro dias de cada período. A massa de ovos foi obtida multiplicando-se o peso médio dos ovos pela porcentagem de produção.

Os dados foram submetidos à análise de variância, utilizando-se o programa SAEG (UNIVERSIDADE FEDERAL DE VIÇOSA, 1982) e as médias, testadas pelo teste de Newman Keuls $(\mathrm{P}<0,05)$. O modelo estatístico usado foi:

em que

$$
Y_{i j}=\mu+t_{i}+e_{i j}
$$

$\mathrm{Y}_{\mathrm{ij}}=$ observação relativa ao i-éssimo tratamento na j-éssima parcela; 
1442 Rev. bras. zootec.

$\mu=$ média de cada característica;

$\mathrm{t}_{\mathrm{i}}=$ efeito do $\mathrm{i}$-éssimo tratamento, sendo $\mathrm{i}=$ $1, \ldots, 4 ; \mathrm{e}$

$\mathrm{e}_{\mathrm{ij}}=$ erro aleatório associado a cada parcela experimental, sendo o erro $\operatorname{NID}\left(0 ; \sigma^{2}\right)$.

\section{Resultados e Discussão}

Desempenho das aves durante a muda forçada

A produção e a massa de ovos foram maiores $(\mathrm{P}<0,01)$ para o grupo de aves no tratamento controle, enquanto os grupos de aves dos tratamentos de muda não diferiram entre si (Tabela 1 ). $O$ peso dos ovos não foi afetado $(\mathrm{P}>0,05)$ pelos tratamentos.

$\mathrm{O}$ uso do carbonato de cálcio durante a muda resultou em produção, peso e massa dos ovos, respectivamente, de 16,$1 ; 2,8$; e $28,9 \%$ a mais que o tratamento com fosfato bicálcico e 2,9; 6,2; e 9,5\% a mais que o tratamento de muda forçada sem fontes de cálcio (Tabela 1).

A análise diária mostrou similar declínio da produção de ovos do primeiro para o terceiro dia em todos os tratamentos de muda forçada (Figura 1), ou seja, o fornecimento de fontes de cálcio não retardou a taxa de declínio de produção de ovos durante a muda forçada. Portanto, este resultado discorda daqueles obtidos por GARLICH e PARKHURST (1982), em que o uso de farinha de ostras como fonte de cálcio retardou a taxa de declínio da produção de ovos durante a muda forçada, em relação ao tratamento controle.

O peso médio das cascas e a porcentagem de casca foram melhores para os grupos de aves dos tratamentos controle e com carbonato de cálcio, em comparação aos resultados obtidos no tratamento de muda sem fontes de cálcio e no tratamento com fosfato bicálcico (Tabela 2).

$\mathrm{O}$ carbonato de cálcio aumentou, em valores percentuais, os ovos disponíveis para a venda e reduziu os ovos perdidos por avaria de casca em relação aos tratamentos de muda sem fontes de cálcio e de muda com fosfato bicálcico durante o período de jejum (Tabela 2), provavelmente, por estimular maior taxa de ovulação nos três primeiros dias da muda forçada e melhor qualidade da casca, mas não em função da extensão da produção de muda, como foi observado por GARLICH e

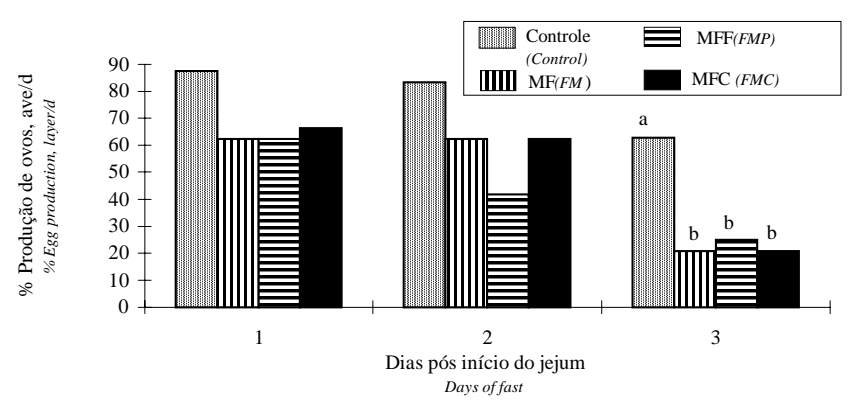

Figura 1 - Produção de ovos do tratamento controle e dos tratamentos de muda forçada (MF), MF mais fosfato bicálcico (MFF) à vontade e MF mais carbonato de cálcio (MFC) à vontade nos dias 1, 2 e 3 do início da retirada da ração.

Figure 1 - Egg production of control and treatments forced molt (FM), FM and dicalcium phosphate ad libitum (FMP), FM and calcium carbonate ad libitum (FMC) in days 1 , 2 , and 3 without diets.

Tabela 1 - Efeito da fonte de cálcio sobre produção de ovos, massa de ovos e peso dos ovos de poedeiras leves durante a muda forçada

Table 1 - Effect of calcium source on egg production, egg mass, and egg weight of whiteegg layers during the forced molt

\begin{tabular}{lccc}
\hline Tratamento $^{1}$ & $\begin{array}{c}\text { Prod. ovos }(\%) \\
\text { Treatments }^{1}\end{array}$ & $\begin{array}{c}\text { Massa de ovos }(\mathrm{g}) \\
\text { Egg mass }\end{array}$ & $\begin{array}{c}\text { Peso do ovo }(\mathrm{g}) \\
\text { Egg weight }\end{array}$ \\
\hline Controle $($ Control) & $77,86^{\mathrm{a}}$ & $47,46^{\mathrm{a}}$ & $61,08^{\mathrm{a}}$ \\
MF $(F M)$ & $48,61^{\mathrm{b}}$ & $26,39^{\mathrm{b}}$ & $54,45^{\mathrm{a}}$ \\
MFF $(F M P)$ & $43,06^{\mathrm{b}}$ & $22,42^{\mathrm{b}}$ & $56,27^{\mathrm{a}}$ \\
MFC $(F M C)$ & $50,00^{\mathrm{b}}$ & $28,91^{\mathrm{b}}$ & $57,83^{\mathrm{a}}$ \\
\hline CV, $\%$ & 12,87 & 14,20 & 9,72 \\
\hline Significância(Significance) & $\mathrm{P}<0,01$ & $\mathrm{P}<0,01$ & $\mathrm{~ns}$ \\
\hline
\end{tabular}

a,b Médias, na coluna, seguidas de letras diferentes diferem pelo teste Newman Keuls.

1 Controle = sem muda; MF = muda forçada; MFF = muda forçada mais fosfato bicálcico; MFC = muda forçada mais carbonato de cálcio.

$a, b$ Means, within a column, followed by different letters differ by Newman Keuls test.

1 Control $=$ without forced molt; FM = forced molt; FMP = forced molt and dicalcium phosphate; $F C C=$ forced molt and calcium carbonate. 
Tabela 2 - Efeito da fonte de cálcio sobre peso da casca, porcentagem de casca, ovos comerciais $(\% \mathrm{OC})$ e ovos perdidos $(\% \mathrm{OP})$ de poedeiras leves durante a muda forçada

Table 2 - Effect of calcium source on egg shell weight, shell percentage, marketable eggs and egg loss of white-egg layers during the forced molt

\begin{tabular}{lcccc}
\hline Tratamento $^{1}$ & $\begin{array}{c}\text { Peso da casca }(\mathrm{g}) \\
\text { Shell weight }\end{array}$ & $\begin{array}{c}\text { \% Casca } \\
\text { shell }\end{array}$ & $\begin{array}{c}\text { \% Ovo comercial } \\
\text { Marketable egg }\end{array}$ & $\begin{array}{c}\text { \% Ovo perdido } \\
\text { Eggs loss }\end{array}$ \\
\hline Controle $($ Control) & $5,32^{\mathrm{a}}$ & $8,70^{\mathrm{a}}$ & 98,61 & 1,39 \\
MF $(F M)$ & $3,36^{\mathrm{b}}$ & $6,18^{\mathrm{b}}$ & 80,00 & 20,00 \\
MFF $(F M P)$ & $3,42^{\mathrm{b}}$ & $6,63^{\mathrm{b}}$ & 72,22 & 27,75 \\
MFC $(F M C)$ & $4,91^{\mathrm{a}}$ & $8,50^{\mathrm{a}}$ & 90,28 & 9,72 \\
\hline CV $\%$ & 10,99 & 6,87 & 18,75 & $\mathrm{n}$ \\
\hline Significância & $\mathrm{P}<0,01$ & $\mathrm{P}<0,01$ & $\mathrm{~ns}$ & \\
Significance & & & &
\end{tabular}

a,b Médias, na coluna, seguidas de letras diferentes diferem pelo teste Newman Keuls.

1 Controle = sem muda; MF = muda forçada; MFF = muda forçada mais fosfato bicálcico; $M F C=$ muda forçada mais carbonato de cálcio.

a,b Means, within a column, followed by different letters differ by Newman Keuls test.

1 Control = without forced molt; $F M=$ forced molt; FMP = forced molt and dicalcium phosphate; $F C C=$ forced molt and calcium carbonate.

PARKHURST (1982). A hipótese de BRAKE (1993) de que a deficiência de cálcio celular é o primeiro fator limitante da ovulação em galinhas em jejum e que o fornecimento de carbonato de cálcio prolonga a ovulação durante os estádios iniciais da muda forçada, retardando a cessação da postura, não foi confirmada no presente estudo.

As análises diárias mostraram que, a partir do segundo dia de muda forçada, as aves do tratamento controle apresentaram melhores peso dos ovos, peso de casca e porcentagem de cascas dos ovos que os grupos de aves submetidas à muda forçada, Figuras 2, 3 e 4, respectivamente.

Entre os tratamentos de muda forçada, o fornecimento de carbonato de cálcio melhorou o peso da casca nos dias 2 e 3 e a porcentagem de casca no dia 3 , em comparação com o tratamento sem fontes de cálcio, e melhorou o peso da casca no dia 2 e a porcentagem de casca no dia 3 , em relação ao tratamento com fosfato bicálcico (Figuras 3 e 4).

O peso dos ovos (Tabela 1) não foi alterado, mas o peso médio e a porcentagem de casca do ovo reduziram $(\mathrm{P}<0,05)$ nos tratamentos de muda com fosfato bicálcico e sem o fornecimento de fontes de cálcio, em relação ao uso do carbonato de cálcio (Tabela 2). Entretanto, o uso do fosfato bicálcico não diferiu do tratamento sem fontes de cálcio (Tabela 2). Este último resultado sugere que a oferta de fosfato bicálcico durante a muda forçada, como fonte de cálcio, não tem efeito na promoção da qualidade da casca dos ovos. Oreduzido consumo de fosfato bicálcico de $0,83 \mathrm{~g} / \mathrm{ave} / \mathrm{dia}$, em relação ao consumo do carbonato de cálcio de 3,05 g/ave/dia, que resultou, respectiva-

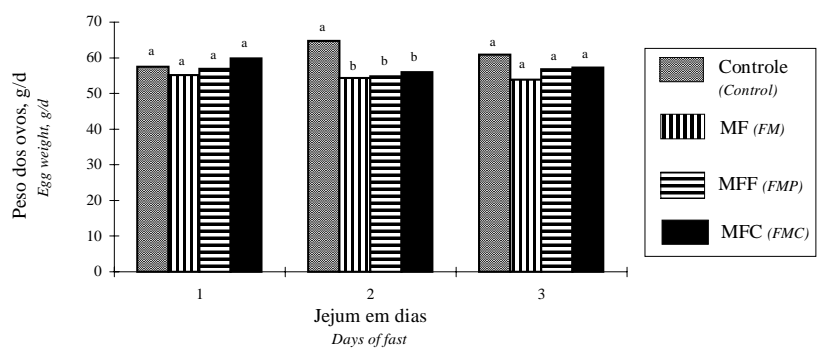

Figura 2 - Peso dos ovos do tratamento controle e dos tratamentos de muda forçada (MF), MF mais fosfato bicálcico (MFF) à vontade e MF mais carbonato de cálcio (MFC) à vontade nos dias 1 , 2 e 3 do início da retirada da ração.

Figure 2 - Egg weight of control and treatments forced molt (FM), FM and dicalcium phosphate ad libitum (FMP), FM and calcium carbonate ad libitum (FMC) in days 1, 2, and 3 without diets.

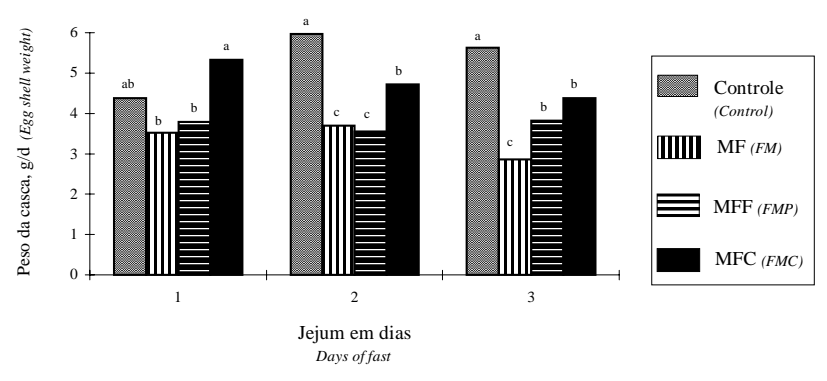

Figura 3 - Pesos da casca dos ovos do tratamento controle e dos tratamentos de muda forçada (MF), MF mais fosfato bicálcico (MFF) à vontade e MF mais carbonato de cálcio (MFC) à vontade nos dias 1 , 2 e 3 do início da retirada da ração.

Figure 3 - Egg shell weight of control and treatments forced molt (FM), FM and dicalcium phosphate ad libitum (FMP), FM and calcium carbonate ad libitum (FMC) in days 1 , 2 , and 3 without diets. 
1444 Rev. bras. zootec.

Tabela 3 - Efeito da fonte de cálcio sobre produção de ovos, peso dos ovos, porcentagem de ovos comerciais e ovos perdidos de poedeiras de ovos brancos após o jejum

Table 3 - Effect of calcium source on the egg production, egg weight, marketable eggs, percentage and egg loss percentage of white-egg layers after fast

\begin{tabular}{lcccc}
\hline $\begin{array}{l}\text { Tratamento }^{1} \\
\text { Treatment }^{1}\end{array}$ & $\begin{array}{c}\text { \% Produção de ovo } \\
\text { Egg production }\end{array}$ & $\begin{array}{c}\text { Peso dos ovo (g) } \\
\text { Egg weight }\end{array}$ & $\begin{array}{c}\text { \% Ovo comercial } \\
\text { Marketable egg }\end{array}$ & $\begin{array}{c}\text { \% Ovo perdido } \\
\text { Egg loss }\end{array}$ \\
\hline Controle $($ Control) & $78,28^{\mathrm{b}}$ & 59,96 & $87,42^{\mathrm{b}}$ & $12,58^{\mathrm{a}}$ \\
MF $(F M)$ & $81,43^{\mathrm{ab}}$ & 61,84 & $98,19^{\mathrm{a}}$ & $1,81^{\mathrm{b}}$ \\
MFF $(F M P)$ & $78,88^{\mathrm{b}}$ & 59,96 & $97,78^{\mathrm{a}}$ & $2,22^{\mathrm{b}}$ \\
MFC $(F M C)$ & $84,92^{\mathrm{a}}$ & 59,53 & $97,08^{\mathrm{a}}$ & $2,92^{\mathrm{b}}$ \\
\hline CV,\% & 3,12 & 2,74 & 4,82 & 93,81 \\
\hline Significância & $\mathrm{P}<0,01$ & $\mathrm{~ns}$ & $\mathrm{P}<0,01$ & $\mathrm{P}<0,01$ \\
Significance & & & & \\
\hline
\end{tabular}

a,b Médias, na coluna, seguidas de letras diferentes diferem pelo teste Newman Keuls.

1 Controle = sem muda; MF = muda forçada; MFF = muda forçada mais fosfato bicálcico; MFC = muda forçada mais carbonato de cálcio.

a,b Means, within a column, followed by different letters differ by Newman Keuls test.

1 Control = without forced molt; FM = forced molt; FMP = forced molt and dicalcium phosphate; FCC = forced molt and calcium carbonate.

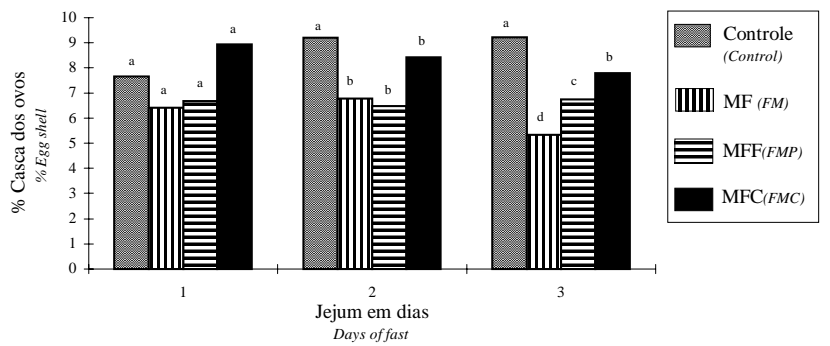

Figura 4 - Percentual de casca dos ovos do tratamento controle e dos tratamentos de muda forçada (MF), MF mais fosfato bicálcico (MFF) à vontade e MF mais carbonato de cálcio (MFC) à vontade nos dias 1,2 e 3 do início da retirada da ração.

Figure 4 - Egg shell percentage of control and treatments forced molt (FM), FM and dicalcium phosphate ad libitum (FMP), FM and calcium carbonate ad libitum (FMC) in days 1,2, and 3 without diets.

mente, em 0,19 e 1,17g de cálcio/ave/dia, foi a principal causa da pior qualidade da casca dos ovos motivada, provavelmente, pela baixa palatabilidade do fosfato bicálcico, quando fornecido de forma exclusiva às aves.

Apesar de vários fatores dificultarem a estimativa da necessidade de cálcio para poedeiras durante o ciclo normal de produção de ovos, ROLAND (1986) sugeriu consumo diário de cálcio de $3,75 \mathrm{~g}$, enquanto o NATIONAL RESEARCH COUNCIL - NRC (1994) recomenda consumo de 3 a 4,5 g de cálcio/ave/dia para obtenção de melhor qualidade da casca dos ovos. Entretanto, durante a fase de jejum, observou-se diminuição gradual da taxa de postura, o que deve ter influenciado a necessidade de cálcio. No presente experimento, o consumo de cálcio a partir do carbonato de cálcio, embora inferior ao recomendado para o nível normal de produção, foi mais adequado que o permitido pelo fosfato bicálcico.

Como o cálcio, segundo MATEOS (1991), constitui cerca de $98 \%$ dos minerais (90\% da casca) presentes na casca do ovo, fica claro que os efeitos negativos do baixo consumo de cálcio são a melhor explicação para a inferior qualidade da casca dos ovos obtida nos tratamentos de muda sem o uso de fonte de cálcio e com o uso do fosfato bicálcico à vontade.

\section{Desempenho pós-muda forçada}

No período posterior à retirada da ração, o tratamento com carbonato de cálcio promoveu maiores produção de ovos e percentual de ovos comerciais e menor percentual de ovos perdidos por problemas de casca em comparação com o controle $(\mathrm{P}<0,01)$, contudo, o peso dos ovos não sofreu efeito dos tratamentos (Tabela 3).

Entre os tratamentos de muda, a produção de ovos foi prejudicada pelo uso do fosfato bicálcico em relação ao de carbonato de cálcio $(\mathrm{P}<0,01)$, que por sua vez não diferiu do tratamento sem fontes de cálcio $(\mathrm{P}>0,05)$. Todavia, em termos percentuais, o carbonato de cálcio acarretou aumento da produção de ovos de 8,$5 ; 4,3$; e $7,7 \%$ a mais que, respectivamente, os tratamentos controle, sem fonte de cálcio e fosfato bicálcico.

Os resultados sugerem que o uso do carbonato de cálcio, durante a muda forçada, deve ser economicamente promissor em relação aos demais tratamentos, pois o efeito positivo sobre o desempenho das aves durante a fase de retirada da ração se estendeu ao novo ciclo de produção. 
Uma hipótese para explicar este resultado seriam a maior conservação das reservas do tecido medular e a menor reabsorção das reservas de cálcio do tecido ósseo cortical para garantir a produção durante a fase de jejum.

\section{Conclusões}

O fornecimento de carbonato de cálcio durante a muda forçada melhora a qualidade da casca dos ovos em comparação ao uso do fosfato bicálcico e ao tratamento sem fonte de cálcio, aumentando substancialmente o número de ovos para a comercialização. No período posterior à muda forçada, o carbonato de cálcio aumenta a produção de ovos, mas as razões para este resultado não são claras.

\section{Referências Bibliográficas}

BELL, D.J., SILLER, W.G. 1962. Cage layer fatigue in Brown Leghorns. Res. Vet. Sci., 3:219-230.

BRAKE, J.T. 1993. Recent advances in molt. Poult. Sci., 72:929-931.

GARLICH, J.D. The phosphorus requirements of laying hens. In: GEORGIA NUTRITION CONFERENCE, Atlanta, 1979. Proceedings... Atlanta, 1979. p.104-114.

GARLICH, J.D., PARKHURST, C.R. 1982. Increased egg production by calcium supplementation during the initial period of a forced molt. Poult. Sci., 61:955-961.
GILBERT, A.G. 1969. The effect of a foreign object in the shell gland on egg production of hens on a calcium deficient diet. Br. Poult. Sci., 10:83-88.

MATEOS, G.C. 1991. Fatores que influyem en la calidad del huevo. In: MATEO, G.C.(Ed.) Nutrición y alimentación de las gallinas ponedoras. 9.ed. Madrid: Mundi Prensa. p.227-248.

MIKAELIN, K.S., SHELL, J.L. 1981. Performance of laying hens fed various phosphorus levels continuously or phase fed detrimental phosphorus levels. Poult. Sci., 60:1916-1926.

MORRIS, T.R., NALBANDOV, A.V. 1961. The induction of ovulation in starging pullets using mamalian and avian gonadotropins. Endocrinology, 68:687-697.

NATIONAL RESEARCH COUNCIL - NRC. 1994. Nutrient requirement of poultry. 9.ed. Washington, D.C.: National Academic Press. 155p.

ROLAND, D.A.Sr. 1986. Egg shell quality III: Calcium and phosphorus requirements of commercial leghorns. World's Poult. Sci. J., 42:154-165.

URIST, M.R. 1959. The effects of calcium deprivation upon the blood, adrenal cortex, ovary and skeleton in the domestic fowl. Rec. Progr. Horm. Res., 15:455-481.

UNIVERSIDADE FEDERAL DE VIÇOSA - UFV. 1982. Central de Processamento de Dados - UFV/CPD. SAEG - Sistema de análises estatísticas e genéticas. Viçosa, MG. 52p.

Recebido em: 05/10/99

Aceito em: 31/02/00 